\title{
Correspondance
}

\section{The ethics of research involving Canada's Aboriginal populations}

$\mathrm{T}$

he recent publication of 2 epidemiologic studies examining Aboriginal populations ${ }^{1,2}$ raises ethical concerns. Neither study describes any consultations with First Nations or Métis community members in the formulation of the research questions, development of the research protocols, interpretation of the data or dissemination of the results. This apparent lack of consultation is particularly problematic given that both studies could have policy implications that would affect First Nations and Métis individuals and communities. A related ethical and methodologic concern is raised by the use of "Aboriginality" as a risk factor in the multivariate analyses of both studies.

As a health research scientist trained in the Western academic disciplines of medicine and public health and as a Métis woman, I respect the efforts of these authors to produce much-needed information on the health of First Nations and Métis peoples. However, I have come to understand that it is only through an approach of mutual understanding, respect and partnership that academic research will be able to contribute to improving the health outcomes in First $\mathrm{Na}$ tions, Métis and Inuit communities..$^{3-8}$

"Aboriginality" is a social construct with little grounding in the day-to-day realities of the heterogeneous groups to which it refers. Tremendous cultural, historical, socioeconomic and political diversity exists between and within these groups. What is shared is the experience of colonization and the resultant legacy of poverty and social stressors. Use of this pan-ethnic term as one of several "risk" variables, while perhaps necessary to achieve adequate study power, devalues the unique experiences of First Nations, Métis and Inuit communities and perpetuates colonial processes of marginalizing or "pathologizing" Aboriginal peoples.

Methodologically, the use of the "Aboriginal" variable in the study by
Tonelli and associates ${ }^{1}$ is problematic. Cox regression analysis assumes that the effects of different variables on survival are constant over time and are additive, but I am not sure that these assumptions are met with respect to the "Aboriginality" factor. Rather, there may be an interaction or multiplicative effect between "Aboriginality" and socioeconomic status, for example. In the study by Wenman and colleagues ${ }^{2}$ there are methodologic problems with the way in which ethnicity was determined. In addition, multiple-ethnicity responses, such as Caucasian and First Nations, were excluded, and the study was underpowered because the sample of First Nations and Métis women was insufficient.

The editorial by Alan Cass ${ }^{9}$ does not address the ethical issues raised by the apparent lack of Aboriginal consultation or the ethical problems relating to the use of the pan-ethnic "Aboriginal" category as a risk factor for disease.

If "Aboriginality" is the factor that these scientists are interested in understanding, I suggest that they start by building relationships with one or more First Nations, Métis or Inuit communities. They may be pleasantly surprised by how quickly this approach will provide new insights and perspectives regarding Aboriginal health and medicine more generally.

\section{Janet Smylie}

Director, Indigenous Peoples Health

Research Centre

Associate Professor

Department of Community Health and Epidemiology

University of Saskatchewan

Saskatoon, Sask.

\section{References}

1. Tonelli M, Hemmelgarn B, Manns B, Pylypchuk G, Bohm C, Yeates K, et al. Death and renal transplantation among Aboriginal people undergoing dialysis. CMA7 2004;171(6):577-82.

2. Wenman WM, Joffres MR, Tataryn IV, Edmonton Perinatal Infections Group. A prospective cohort study of pregnancy risk factors and birth outcomes in Aboriginal women. CMAJ 2004;171(6):585-9.

3. KSDPP code of research ethics. Kahnawake Territory, Mohawk Nation (QC): Kahnawake Schools Diabetes Prevention Project; [date unknown]. Available: www.ksdpp.org/code.html (accessed 2004 Nov 17).

4. Royal Commission on Aboriginal Peoples. Appendix E: Ethical guidelines for research. In: $R e-$ port of the Royal Commission on Aboriginal Peoples. Vol 5. Renewal: a twenty-year commitment. Ottawa: The Commission; 1996, updated 2004 Apr 23. Available: www.ainc-inac.gc.ca/ch/rcap /sg/sgmm_e.html (accessed 2004 Nov 17)

5. Inuit Tapirisat of Canada. Negotiating research relationships: a guide for communities. Ottawa: Nunavut Research Institute, Inuit Tapirisat of Canada; 1998.

6. Masuzumi B, Quirk S. Dene tracking. A participatory research process for Dene/Metis communities: exploring community-based research concerns for Aboriginal northerners. Yellowknife: Dene Nation; 1993.

7. Tri-council policy statement. Ethical conduct for research involving humans. Ottawa: Natural Sciences and Engineering Council of Canada, Medical Research Council of Canada, Social Sciences and Humanities Research Council of Canada; 1998. Available: www.ncehr-cnerh .org/english/code_2/ (accessed 2004 Nov 17).

8. United Nations Working Group on Indigenous Populations. 1994/45 draft United Nations declaration on the rights of indigenous peoples. Geneva: United Nations; 1994. E/CN.4/Sub.2 /1994/56.

9. Cass A. Health outcomes in Aboriginal populations [editorial]. CMA7 2004;171(6):597-8.

DOI:10.1503/cmaj.1041676

\section{[Dr. Tonelli and 3 coauthors respond:]}

T $\mathrm{T}$ e appreciate this opportunity to describe the involvement of Aboriginal communities in the interpretation and dissemination of our research findings. ${ }^{1}$ We fully agree that relationships with Aboriginal communities are essential for conducting relevant and helpful investigations of the health of 
First Nations, Inuit and Métis people, and we have taken care to build such relationships in the conduct of our work.

The study on death and renal transplantation among Aboriginal people undergoing dialysis ${ }^{1}$ was the first in a planned series of investigations that will include community representation and ongoing input from Aboriginal communities and Aboriginal leaders in $\mathrm{Al}-$ berta and Ontario. These investigations have been approved by the appropriate institutional review boards, and no concerns have been raised about the ethics of the work.

To ensure that our work appropriately addressed the unique situation of Aboriginal people, the final manuscript for this study ${ }^{1}$ was reviewed in detail by 2 First Nations elders, neither of whom expressed the concerns raised by Smylie.

To strengthen our research team (and provide the unique insights to which Smylie refers), we have established ongoing collaboration with leading Aboriginal researchers. Through their involvement, as well as the collaboration and partnership of members of the Aboriginal communities, we are formulating a plan for dissemination of these research results and will suggest changes to health policy accordingly.

Smylie also raises methodological concerns about our study. As stated in our article, ${ }^{1}$ the assumptions of the Cox proportional hazards models were tested and met. We agree that the possibility of an interaction between socioeconomic status and race on health outcomes seems plausible. However, as we reported, this hypothesis was tested and was nonsignificant, which suggests that such an interaction did not exist in the population we studied.

\author{
Marcello Tonelli \\ Department of Medicine \\ Division of Nephrology \\ University of Alberta \\ Edmonton, Alta. \\ Brenda Hemmelgarn \\ Department of Community Health \\ Sciences \\ University of Calgary \\ Calgary, Alta. \\ Karen Yeates
}

Department of Medicine

Division of Nephrology

Queen's University

Kingston, Ont.

John Gill

Division of Nephrology

St. Paul's Hospital

Vancouver, BC

\section{Reference}

1. Tonelli M, Hemmelgarn B, Manns B, Pylypchuk G, Bohm C, Yeates K, et al. Death and renal transplantation among Aboriginal people undergoing dialysis. CMA7 2004;171(6):577-82.

DOI:10.1503/cmaj.1050058

\section{[Dr. Wenman and colleagues respond:]}

Tanet Smylie expresses ethical and $\int$ methodological questions about our study. ${ }^{1}$ In the first instance, it is important to realize that our study did not constitute participatory action research, ${ }^{2}$ which would indeed require what Smylie suggests in terms of involvement of ethnic groups. Rather, as stated in the Methods section, our study involved a hospital-based cohort of pregnant women and did not initially target any specific ethnic group. Data on ethnic origin, among other variables, were collected as part of the study, and demographic and clinical details have been published elsewhere. ${ }^{3}$ The decision to prepare the CMAJ paper for publication arose from our analysis of the data, during which we found important differences between ethnic groups that we thought should be reported because of their public health implications.

Smylie also raises some methodologic concerns. We are unsure what is meant by her reference to "methodologic problems with the way in which ethnicity was determined," since no details are given about where we might have erred. We used standard questions and classified ethnicity according to current standards. Smylie is concerned about the exclusion of "multipleethnicity responses, such as Caucasian and First Nations." Only 3 First Nations and 3 Métis women also checked off Caucasian ethnicity, and these people were not coded as Caucasian because the categories were mutually 